OPEN ACCESS

Edited by:

Billy Sperlich,

University of Würzburg, Germany

Reviewed by:

Christian Brinkmann,

German Sport University Cologne,

Germany

Karsten Müssig,

German Diabetes Center (LG),

Germany

${ }^{*}$ Correspondence:

Jonathan P. Little

jonathan.little@ubc.ca

Specialty section:

This article was submitted to

Exercise Physiology,

a section of the journal

Frontiers in Physiology

Received: 17 May 2017

Accepted: 07 July 2017

Published: 25 July 2017

Citation:

Francois ME, Durrer C, Pistawka KJ,

Halperin FA, Chang $C$ and Little JP

(2017) Combined Interval Training and

Post-exercise Nutrition in Type 2

Diabetes: A Randomized Control Trial.

Front. Physiol. 8:528.

doi: 10.3389/fphys.2017.00528

\section{Combined Interval Training and Post-exercise Nutrition in Type 2 Diabetes: A Randomized Control Trial}

\author{
Monique E. Francois ${ }^{1}$, Cody Durrer ${ }^{1}$, Kevin J. Pistawka ${ }^{2}$, Frank A. Halperin ${ }^{2}$, \\ Courtney Chang ${ }^{1}$ and Jonathan P. Little ${ }^{1 *}$
}

${ }^{1}$ School of Health and Exercise Sciences, University of British Columbia Okanagan, Kelowna, BC, Canada, ${ }^{2}$ Kelowna General Hospital, Kelowna Cardiology Associates, Kelowna, BC, Canada

Background: High-intensity interval training (HIIT) can improve several aspects of cardiometabolic health. Previous studies have suggested that adaptations to exercise training can be augmented with post-exercise milk or protein consumption, but whether this nutritional strategy can impact the cardiometabolic adaptations to HIIT in type 2 diabetes is unknown.

Objective: To determine if the addition of a post-exercise milk or protein beverage to a high-intensity interval training (HIIT) intervention improves cardiometabolic health in individuals with type 2 diabetes.

Design: In a proof-of-concept, double-blind clinical trial 53 adults with uncomplicated type 2 diabetes were randomized to one of three nutritional beverages $(500 \mathrm{~mL}$ skim-milk, macronutrient control, or flavored water placebo) consumed after exercise (3 days/week) during a 12 week low-volume HIIT intervention. HIIT involved $10 \times 1$-min high-intensity intervals separated by 1-min low-intensity recovery periods. Two sessions per week were cardio-based (at $\sim 90 \%$ of heart rate max) and one session involved resistancebased exercises (at RPE of 5-6; CR-10 scale) in the same interval pattern. Continuous glucose monitoring (CGM), glycosylated hemoglobin $\left(\mathrm{HbA}_{1 \mathrm{c}}\right)$, body composition (dualenergy X-ray absorptiometry), cardiorespiratory fitness $\left(\mathrm{V}_{2}\right.$ peak $)$, blood pressure, and endothelial function (\%FMD) were measured before and after the intervention.

Results: There were significant main effects of time (all $p<0.05$ ) but no difference between groups (Interaction: all $p>0.71)$ for CGM 24-h mean glucose $(-0.5 \pm 1.1$ $\mathrm{mmol} / \mathrm{L}), \mathrm{HbA}_{1 \mathrm{c}}(-0.2 \pm 0.4 \%)$, percent body fat $(-0.8 \pm 1.6 \%)$, and lean mass $(+1.1$ $\pm 2.8 \mathrm{~kg})$. Similarly, $\dot{\mathrm{V}} \mathrm{O}_{2 \text { peak }}(+2.5 \pm 1.6 \mathrm{~mL} / \mathrm{kg} / \mathrm{min})$ and \%FMD $(+1.4 \pm 1.9 \%)$ were increased, and mean arterial blood pressure reduced $(-6 \pm 7 \mathrm{mmHg})$, after 12 weeks of HIIT (all $p<0.01$ ) with no difference between beverage groups (Interaction: all $p>0.11$ ).

Conclusion: High-intensity interval training is a potent stimulus for improving several important metabolic and cardiovascular risk factors in type 2 diabetes. The benefits of HIIT are not augmented by the addition of post-exercise protein.

Keywords: dairy, exercise, lifestyle, body composition, glycemic control, endothelial function, blood pressure 


\section{INTRODUCTION}

Worldwide more than 257 million people have type 2 diabetes, a figure projected to reach 395 million by 2030 (Shaw et al., 2010). Of those, $71 \%$ have hypertension and $40 \%$ have three or more coexisting chronic conditions, with cardiovascular disease the leading cause of mortality (Centers for Disease Control and Prevention, 2014). Accordingly, interventions that improve both glycemic control and reduce cardiovascular risk factors are central to reducing the burden of type 2 diabetes (Inzucchi et al., 2012). Lifestyle interventions, including exercise and nutrition are at the forefront for the prevention of diabetes complications (Inzucchi et al., 2012). Intensive glucose lowering with multiple pharmacological treatments leads to reduced microvascular complications (UK Prospective Diabetes Study Group, 1998), but the effect on macrovascular complications is unclear.

Large controlled trials and numerous experimental studies reveal the widespread benefits of exercise for people with type 2 diabetes (Marwick et al., 2009; Lin et al., 2015). The Look AHEAD (Action for Health in Diabetes) trial showed that moderate continuous exercise and a caloric restrictive diet leads to sustained reductions in cardiometabolic risk factors, diabetes complications, and health costs (Wing et al., 2013). However, the number of cardiovascular events between the intervention and control groups was not different. The addition of vigorous exercise may be required to elicit substantial changes in cardiovascular function (Baldi et al., 2016), as it appears that vigorous, but not low-moderate exercise, reduces cardiovascular disease (Tanasescu et al., 2002; Lee et al., 2003). Studies using higher exercise intensities, such as interval and resistance exercise, show strong effects on cardiometabolic outcomes (Wisløff et al., 2007; Weston et al., 2014).

Cardiorespiratory fitness is an independent predictor of allcause mortality and cardiovascular events (Kodama et al., 2009). A recent meta-analysis revealed that the increase in fitness after interval training is $\sim 2$-fold greater than continuous training (Weston et al., 2014). In the longest trial to date comparing interval and continuous exercise in diabetes, Karstoft et al. (2013) randomized participants to 4 months interval walking ( $n=$ $12)$, energy and time-matched continuous walking ( $n=12 ; 60$ $\min , 5$ days/week), or non-exercise control $(n=8)$. Greater improvements in fitness, body fat, and glycemic control were observed after interval compared to continuous walking and control (Karstoft et al., 2013). These findings clearly support the benefit of interval exercise, however the volume of exercise (300 $\mathrm{min} /$ week) is far greater than usually attained by the general population, many of whom cite lack of time as a considerable exercise barrier (Korkiakangas et al., 2009). Emerging evidence from small short-term trials show that low-volume high-intensity

Abbreviations: HIIT, High-intensity interval training; FMD, Flow mediated dilation; $\dot{\mathrm{VO}}_{2 \text { peak }}$, Cardiorespiratory fitness; CGM, Continuous glucose monitoring; MAGE, mean amplitude of glycemic excursions; QoL, Quality of Life; $\mathrm{HR}_{\max }$, Peak heart rate; RPE, Rating of perceived exertion; VAT, Visceral Adipose Tissue. interval training (HIIT) rapidly improves glycemic control in type 2 diabetes (Little et al., 2011; Madsen et al., 2015). Lowvolume HIIT involves alternating brief periods of vigorous exercise with periods of recovery, typically taking $\sim 20$ min per session and performed three times per week (Little et al., 2011). Further research is needed to confirm changes in cardiometabolic health outcomes after several months of low-volume HIIT in studies with larger sample sizes.

Sarcopenic obesity disproportionately affects people with type 2 diabetes (Park et al., 2009). Diminished lean muscle leads to poor physical functioning, glycemic control and cardiovascular health (Anton et al., 2013). The anabolic effects of exercise (Robinson et al., 2017) and high-quality protein (Reitelseder et al., 2011) are important for counteracting the age-associated decline in muscle, and when combined, provide synergistic effects on muscle protein synthesis (Esmarck et al., 2001; Hartman et al., 2007). In particular, it appears that consuming milk-protein after exercise promotes significant lean mass accretion and fat loss (Hartman et al., 2007; Josse et al., 2010). HIIT was recently shown to promote increased protein synthesis in the skeletal muscle of older adults, an effect linked to improved insulin sensitivity and mitochondrial function (Robinson et al., 2017). Combining HIIT with postexercise protein supplementation therefore holds potential for maximizing skeletal muscle adaptations in order to improve cardiometabolic health outcomes, particularly in older adults.

The purpose of this study was to determine whether postexercise milk augments the cardiometabolic benefits of lowvolume HIIT in individuals with type 2 diabetes. The primary outcome of glycemic control was assessed across 3 days before and after the intervention using continuous glucose monitoring (CGM). Secondary outcomes of body composition, $\mathrm{HbA}_{1 \mathrm{c}}$, fasting blood parameters, fitness, blood pressure, and endothelial function were also examined to determine how low-volume HIIT impacted key cardiometabolic health parameters.

\section{RESEARCH DESIGN AND METHODS}

\section{Study Design}

This double-blind, randomized clinical trial was conducted at The University of British Columbia Okanagan between January 2015 and December 2016 (clinicaltrials.gov \#NCT02251301). The Clinical Research Ethics Board (CREB \#H14-01636) approved the study and participants provided written informed consent. Randomization was by a third-party using variable permuted block sizes with computer-generated random numbers and sealed envelopes. A researcher not involved in data analysis prepared the beverages so participants and study personnel were blinded to the beverage condition.

\section{Participants}

Men and women between 40 and 75 years with physiciandiagnosed type 2 diabetes ( $>6$ months) were recruited from the Kelowna Diabetes Program via mail-out advertisements and sign-up sheets. Exogenous insulin users, diagnosed cardiovascular disease and diabetes complications, or contraindications to exercise (Thompson et al., 2013) 
were excluded. After telephone/email interviews interested participants attended a screening visit, which included a medical history questionnaire, physical activity readiness questionnaireplus (PARQ+), and informed consent. Eligible participants then completed a 12-lead stress test using a modified Bruce protocol and a cardiologist provided clearance for vigorous exercise.

\section{Intervention}

\section{Experimental Protocol Overview}

Fifty-three participants were randomized to one of three beverages; (i) low-fat milk, (ii) macronutrient control, or (iii) placebo, consumed after exercise (details below). Baseline and post-intervention outcomes were assessed over 5 days before and after the intervention (48-72 $\mathrm{h}$ after the last training session). Fasted blood and body composition measures were obtained on day 1 and CGM was performed across days 2-4 while participants followed a standardized diet. Blood pressure, endothelial function and fitness were assessed on day 5. Body weight, waist circumference, blood pressure, and endothelial function were also assessed at 6 weeks (Mid).

\section{Exercise Training}

All groups performed supervised low-volume HIIT 3d/week for 12 weeks. To be consistent with exercise recommendations by the American Diabetes Association and the American College of Sports Medicine (Colberg et al., 2016) both resistance and cardio-based exercises were included in the HIIT program. The first and last sessions per week were cardio-based (cycle ergometer, treadmill, or elliptical) involving 1-min bursts of exercise at $85-90 \%$ of the participants' maximum heart rate ( $\mathrm{HR}_{\max }$; obtained during baseline $\dot{\mathrm{VO}}_{2 \text { peak }}$ test) with 1 min of easy recovery in between. The middle session each week involved whole-body resistance exercises (using resistance bands or multigym). Similar to cardio-based HIIT, each resistance exercise was performed for $1 \mathrm{~min}$ (as many repetitions as possible) at an intensity eliciting an RPE of 5 "hard" on the CR-10 scale (Borg, 1962) followed by $1 \mathrm{~min}$ of recovery. A 3-min warm-up and cool-down was performed with all sessions. The number of 1min intervals in each session progressed from four in week one to ten in week six of training. Thereafter, $10 \mathrm{X} \mathrm{1-min} \mathrm{intervals}$ eliciting $\sim 90 \%$ of $\mathrm{HR}_{\max }$ (cardio-based) or RPE $\sim 5$ (resistancebased) were completed in each session. Previous short-term training studies in individuals with, and at risk for, diabetes, have shown this low-volume HIIT protocol is effective for improving cardiometabolic health (Little et al., 2011; Francois et al., 2016). A heart rate monitor was worn to closely prescribe intensity, and capillary blood glucose and blood pressure measures were obtained before and after each exercise session.

\section{Post-exercise Nutrition Supplementation}

After each session participants consumed $500 \mathrm{~mL}$ of either: (i) low-fat milk; (ii) milk protein macronutrient-matched control; or (iii) placebo (water), within $1 \mathrm{~h}$. The beverages were designed to look and taste similar and distributed in opaque containers. To accomplish this, one-teaspoon of cocoa powder and $1 / 4$ teaspoon of stevia (Stevia In The Raw ${ }^{\circledR}$, Cumberland Packing Corp; containing $\sim 28 \mathrm{mg}$ stevia) were added to each beverage. Low-fat milk was prepared from skim-milk powder (MedallionMilk Co., Canada) providing 187 calories, $19 \mathrm{~g}$ protein, $26 \mathrm{~g}$ carbohydrate, and $<1 \mathrm{~g}$ of fat. Macronutrientmatched control (milk protein concentrate; Vitalus Nutrition Inc., Canada plus lactose; NOW ${ }^{\circledR}$ Foods, IL, US) provided 186 calories, $21 \mathrm{~g}$ protein, $24 \mathrm{~g}$ carbohydrate, and $<1 \mathrm{~g}$ of fat; i.e., providing the same macronutrient and protein composition as milk but without the micronutrients and other bioactive factors. The placebo beverage provided $<10$ calories from the cocoa powder.

\section{Outcomes}

\section{Continuous Glucose Monitoring (CGM)}

A continuous glucose monitor (iPro 2, Medtronic Inc.) was used to continuously measure blood glucose across 3 days before and after the intervention. CGM provides valuable insight (that a one-off fasting blood or $\mathrm{HbA}_{1 c}$ sample cannot) into glycemic variability and the magnitude of postprandial excursions across several days under free-living conditions (Klonoff, 2005). The CGM continuously samples interstitial fluid from the abdomen, measuring glucose concentration every 5-min using the glucose oxidase reaction (Rossetti et al., 2010). Participants took capillary glucose samples $(4 \mathrm{X} / \mathrm{d})$, which were used to retrospectively calculate retrospective blood glucose concentration via an algorithm within the online software program (CareLink Pro, Medtronic; Rossetti et al., 2010). All food, drink, and medication were recorded (including time eaten, amount, brand) for pretesting, and then replicated exactly for post-intervention.

The primary outcome was 24-h average glucose (from 00:00 to 23:55), calculated as the mean of the 3 CGM days. Standard deviation of 24-h blood glucose and mean amplitude of glycemic excursions (MAGE; Molnar et al., 1970) were calculated from the same 24 -h periods to assess glycemic variability.

\section{Body Composition}

Waist circumference (WHO Expert Consultation, 2008), height and weight (Seca 700, Hamburg, Deutschland) were measured to the nearest $0.1 \mathrm{~cm}$ and $0.1 \mathrm{~kg}$, respectively. Percent fat, visceral adipose tissue (VAT) and lean body mass (LBM) were measured by dual-energy X-ray absorptiometry (Hologic Discovery DXA, MA, USA). All measures were performed and analyzed by the same researcher, with calibrations and quality control testing performed daily.

\section{Cardiorespiratory Fitness $\left(\mathrm{V}_{2 \text { peak }}\right)$}

$\dot{\mathrm{VO}}_{2 \text { peak }}$ was assessed using a maximal incremental ramp test on a cycle ergometer (Lode Excalibur, Netherlands) with continuous sampling of expired gases (Parvomedics TrueOne2400, USA). Beginning at $30 \mathrm{~W}$, the test increased by $1 \mathrm{~W}$ every $4 \mathrm{~s}(15 \mathrm{~W} / \mathrm{min})$ until volitional exhaustion or contraindication (Fletcher et al., 2013). $\mathrm{VO}_{2 \text { peak }}$ and RER were calculated from the highest 30-s average, while $\mathrm{HR}_{\max }$ was recorded as the highest value obtained during the test.

\section{Biochemical Analyses}

Fasting blood samples were collected by venipuncture into EDTA containing tubes, centrifuged for $15 \mathrm{~min}\left(1,550 \mathrm{~g}\right.$ at $\left.4^{\circ} \mathrm{C}\right)$ and 
the plasma stored at $-80^{\circ} \mathrm{C}$ for subsequent batch analyses. Medications were withheld the morning of the fasting blood sample. Fasting glucose was measured by the hexokinase method, high-sensitivity C-reactive protein by latex particle enhanced immunoturbidimetric assay and triglycerides by the enzymatic glycerol kinase and glycerol phosphate oxidase method. Pre and post-intervention samples were analyzed concurrently in duplicate (average coefficient of variation 6.8\%) on a clinical chemistry analyzer (Chemwell 2910, Awareness Technologies) using assays from Pointe Scientific (MI, USA). $\mathrm{HbA}_{1 c}$ was analyzed from a separate EDTA tube by a medical laboratory that routinely performs this analysis according to the National Glycohemoglobin Standardization Program (NGSP).

\section{Blood Pressure and Endothelial Function}

All measures were assessed $4 \mathrm{~h}$ postprandial, after abstaining from alcohol and caffeine for $12 \mathrm{~h}$ and, within participants, at the same time of day with meal and medication standardized. After $20 \mathrm{~min}$ of rest in a supine position, blood pressure was measured manually using the auscultatory method, at least twice to the nearest $2 \mathrm{mmHg}$.

\section{Flow-mediated dilation}

Brachial artery flow-mediated dilation (FMD) is an important prognostic indicator of endothelial function and incident cardiovascular disease (Yeboah et al., 2007). The ability of the vessel to dilate (\%FMD) is measured in response to a physiological (shear stress) stimulus (Thijssen et al., 2011). In the current study, brachial artery FMD was assessed according to current guidelines (Thijssen et al., 2011). Briefly, simultaneous measures of diameter and blood velocity were obtained with high-resolution ultrasound (Terason 3200), $2 \mathrm{~cm}$ from the antecubital fossa. Data were collected over a 1-min baseline, for the last $30 \mathrm{~s}$ of a $5 \mathrm{~min}$ period of forearm ischemia (pneumatic cuff inflated $60 \mathrm{mmHg}$ above systolic blood pressure) and for $3 \mathrm{~min}$ thereafter.

\section{Brachial artery dilatory capacity}

The peak blood flow and diameter response to ischemic handgrip exercise provides an index of resistance artery size or remodeling and the maximal dilatory capacity (Naylor et al., 2005). This is important since changes in artery function (\%FMD) with exercise training are thought to occur rapidly (i.e., first few weeks) after which are superseded by changes in structure, potentially concealing further changes in function (Tinken et al., 2010). After 15 min of rest, following the FMD procedure, baseline diameter and blood velocity were recorded for $1 \mathrm{~min}$. This was followed by $5 \mathrm{~min}$ of forearm ischemia (as above), including $3 \mathrm{~min}$ of isotonic handgrip exercise ( 1 contraction every $2 \mathrm{~s}$ using a dynamometer) between 1-min periods of ischemia alone (Naylor et al., 2005). Again recording resumed $30 \mathrm{~s}$ before cuff deflation and continued for 3 min thereafter.

Absolute FMD (peak diameter - baseline diameter), \%FMD (peak - baseline diameter/baseline diameter), and time to peak diameter were measured using custom designed edgedetection and wall-tracking software, which minimizes user bias (Woodman et al., 2001). This protocol is routinely performed
TABLE 1 | Baseline characteristics of participants.

\begin{tabular}{|c|c|c|c|}
\hline & Milk $(n=18)$ & $\begin{array}{l}\text { Macronutrient } \\
\text { control }(n=16)\end{array}$ & $\begin{array}{l}\text { Placebo } \\
(n=19)\end{array}$ \\
\hline Sex & $11 \mathrm{~F}$ & $12 \mathrm{~F}$ & $11 \mathrm{~F}$ \\
\hline Age $(y)$ & $62 \pm 8$ & $56 \pm 9$ & $55 \pm 9$ \\
\hline $\mathrm{BMI}\left(\mathrm{kg} / \mathrm{m}^{2}\right)$ & $36 \pm 7$ & $35 \pm 6$ & $33 \pm 6$ \\
\hline Years of diagnosis & $6 \pm 6$ & $7 \pm 7$ & $5 \pm 6$ \\
\hline \multicolumn{4}{|l|}{ MEDICATIONS } \\
\hline Lifestyle only & 5 & 5 & 3 \\
\hline Metformin & 10 & 11 & 13 \\
\hline Sulfonylureas & 6 & 1 & 3 \\
\hline SGLT2 inhibitors & 1 & 2 & 3 \\
\hline DPP4 inhibitors & 1 & 2 & 3 \\
\hline GLP1 analogs & 1 & 2 & 0 \\
\hline Lipid lowering & 9 & 7 & 7 \\
\hline Antihypertensive & 7 & 6 & 8 \\
\hline \multicolumn{4}{|c|}{ BASELINE PHYSICAL ACTIVITY } \\
\hline LTPA score & $17 \pm 15$ & $14 \pm 10$ & $21 \pm 17$ \\
\hline MVPA (min/day) & $14 \pm 15$ & $13 \pm 13$ & $30 \pm 19$ \\
\hline Dairy intake (servings/day) & $2.3 \pm 2.4$ & $2.7 \pm 2.1$ & $2.1 \pm 1.6$ \\
\hline
\end{tabular}

F, Females; LTPA, Leisure-Time Physical Activity; MVPA, Moderate-Vigorous Physical Activity.

in our lab using the methods outlined in Francois et al. (2016); coefficients of variation for diameter and \%FMD are 2.1 and $7.3 \%$, respectively.

\section{Quality of Life (QoL)}

Participants completed the Medical Outcomes Study Short Form 36 (SF-36) questionnaire before and after the intervention (McHorney et al., 1994). The SF-36 is a self-report QoL questionnaire; the scores are used to provide two norm-based $\mathrm{T}$ scores, physical component summary (PCS) and mental component summary (MCS).

\section{Diet and Exercise Standardization}

Participants maintained their usual diet, lifestyle, and medication habits throughout the testing and training sessions, verified by physical activity and diet records. Baseline dairy consumption was assessed using a food frequency questionnaire, and dietary intake before and during the study was assessed using 3day diet records analyzed using FoodWorks16 (The Nutrition Company, NJ, USA). Baseline activity was examined using both accelerometry (Actigraph GT3x, FL, USA) over a 7-day period to assess minutes of moderate-vigorous physical activity (MVPA, Freedson et al., 1998 cut-points) and a Godin leisure-time exercise questionnaire (Godin and Shephard, 1997; Table 1).

\section{Statistical Analyses Sample Size}

Using means and standard deviations from previously published data on the change in CGM assessed hyperglycemia in type 2 diabetes after HIIT (Little et al., 2011), power calculations determined that $n=17$ per group would be sufficient to detect 
a $30 \%$ reduction in glucose (Cohen $d=0.7$ ) with a power of $80 \%$ and alpha of 0.05 .

\section{Statistics}

Analyses were performed on all participants that completed the intervention. Characteristics of the intervention groups are shown in Table 1. Linear mixed models using SPSS 22.0 (SPSS, Chicago, Illinois) examined changes in trial outcomes (prepost or pre-mid-post) between groups. Significant interactions were probed with pre-planned contrasts comparing the change within each group, whereas isolated significant main effects of time were examined by pairwise comparisons with groups collapsed using Least Significant Difference (LSD) test (Hopkins et al., 2009). Results are reported as means and standard deviations with 95\% confidence limits. Magnitude based inferences were used to identify clinically meaningful changes in major outcomes using techniques described by Batterham and Hopkins (2006). The threshold for clinically beneficial changes in 24-h glucose and $\mathrm{HbA}_{1 c}$ were reductions of 0.5 $\mathrm{mmol} / \mathrm{l}$ and $0.7 \%$, respectively, based on the reduced risk for diabetes complications (Mazzone, 2010). For cardiorespiratory fitness an increase of 1 metabolic equivalent (MET) was used for a $15 \%$ risk reduction in cardiovascular disease (Kodama et al., 2009). For \%FMD $+1 \%$ was used, based on the $13 \%$ risk reduction in cardiovascular events (Inaba et al., 2010). In line with previous studies, a $2 \mathrm{mmHg}$ reduction in MAP was considered to be the smallest clinical threshold change for BP (Cook et al., 1995). The clinically meaningful difference in QOL was determined as a change $>3$ points (Warkentin et al., 2014).

\section{RESULTS}

\section{Participant Compliance and Adverse Events}

Figure 1 shows the CONSORT flow diagram of study progression. Fifty-three participants were eligible after screening; four required additional 24-h blood pressure monitoring $(n=$ 2 ) and stress echo $(n=2)$ cardiologist clearance following the 12-lead ECG stress test. Baseline characteristics of randomized participants are shown in Table 1. The majority (51/53) were of European descent, while two were Southeast Asian $(2 / 53)$.

Of the 53 participants randomized, 51 successfully completed 36 sessions of HIIT in $12 \pm 1 \mathrm{wk}$. One participant suffered a nonfatal myocardial infarction in week eight (after 23 HIIT sessions) and one dropped out for personal reasons. There were no reports of hypoglycemia after exercise or at home throughout the intervention. Exercise sessions were rescheduled on 10 occasions ( $n=6$ due to sickness and $n=4$ due to systolic blood pressure $>144 \mathrm{mmHg}$ prior to exercise). No musculoskeletal injuries were reported as a result of the training. $\dot{\mathrm{V}} \mathrm{O}_{2 \text { peak }}$ testing was truncated in three participants because systolic pressure exceeded 250 mmHg during the test (Fletcher et al., 2013). For CGM analyses three participants were excluded due to sensor failure $(n=1)$ and medication changes ( $n=2$; required reduced medication). All other analyses are reported for $n=51$ unless otherwise
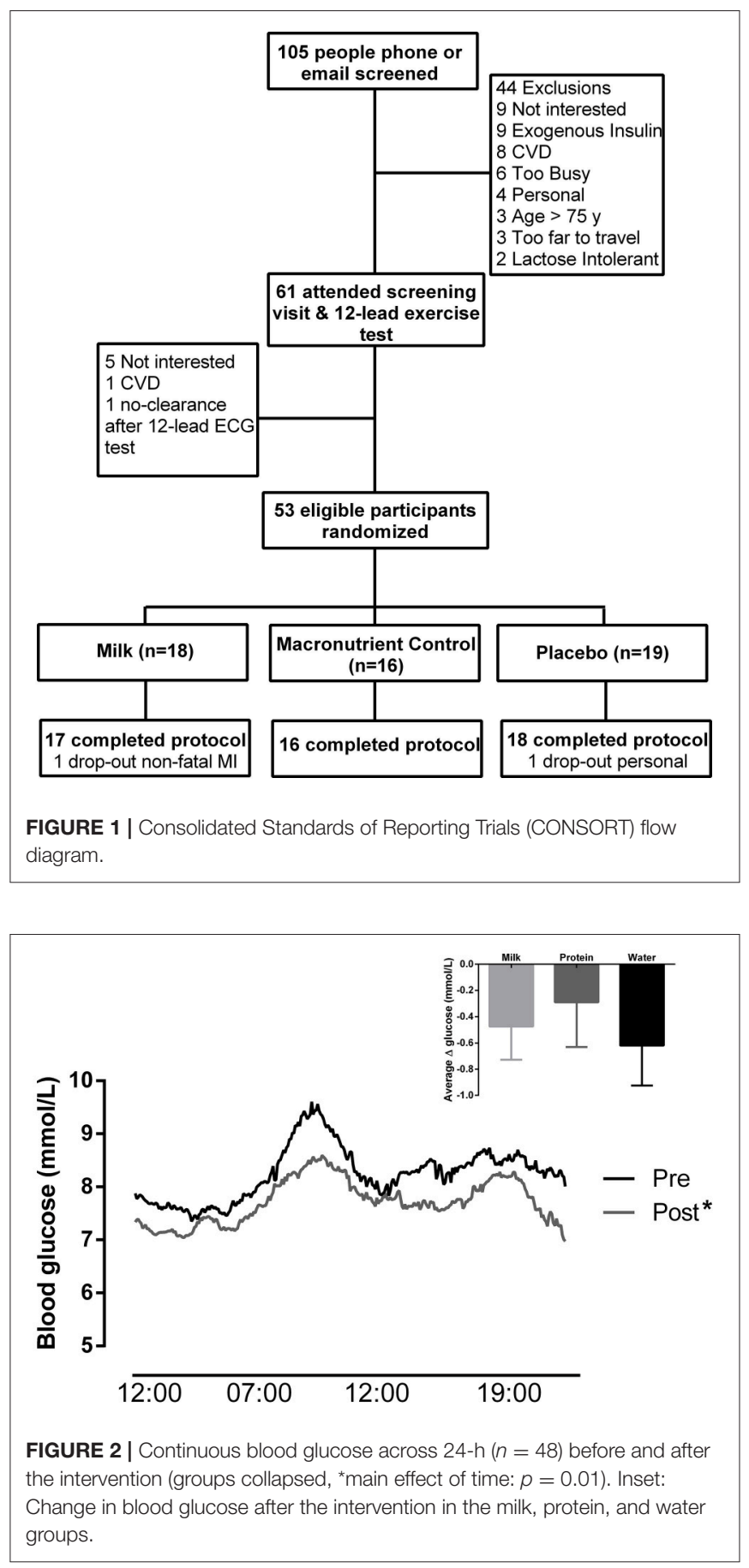

stated. Overall the exercise intensity achieved was $88 \pm 7 \%$ of $\mathrm{HR}_{\max }$ during cardio-based intervals, and an average RPE of $5 \pm 1$ and $4 \pm 1$, for cardio- and resistance-based intervals, respectively.

\section{Glycemic Control}

There was a significant reduction in mean 24-h glucose following 12 weeks of HIIT (by $-0.5 \pm 1.1 \mathrm{mmol} / \mathrm{L}$, Figure 2) with no difference between groups (Table 2). The probability that the 
TABLE 2 | Body composition, cardiorespiratory fitness, blood pressure, flow-mediated dilation, triglycerides, C-reactive protein, and glycemic control measures before and after 12 weeks of HIIT and nutritional beverage.

\begin{tabular}{|c|c|c|c|c|c|c|c|c|}
\hline & \multicolumn{2}{|c|}{ Milk $(n=18)$} & \multicolumn{2}{|c|}{ Macronutrient control $(n=16)$} & \multicolumn{2}{|c|}{ Placebo $(n=19)$} & \multicolumn{2}{|c|}{$P$-value } \\
\hline & Pre & Post & Pre & Post & Pre & Post & Interaction & Time \\
\hline \multicolumn{9}{|l|}{ BODY COMPOSITION } \\
\hline Mass (kg) & $97.7 \pm 19.3$ & $96.8 \pm 20.5$ & $95.9 \pm 17.3$ & $94.5 \pm 17.3$ & $89.5 \pm 21.1$ & $89.1 \pm 20.9$ & 0.46 & $0.03^{\star}$ \\
\hline VAT $(g)$ & $1057 \pm 335$ & $1033 \pm 316$ & $1007 \pm 260$ & $981 \pm 212$ & $815 \pm 337$ & $802 \pm 285$ & 0.75 & 0.25 \\
\hline \multicolumn{9}{|c|}{ CARDIORESPIRATORY FITNESS } \\
\hline$\dot{\mathrm{V}} \mathrm{O}_{2 \text { peak }}(\mathrm{L} / \mathrm{min})$ & $1.7 \pm 0.4$ & $2.0 \pm 0.7$ & $1.8 \pm 0.5$ & $2.0 \pm 0.6$ & $1.9 \pm 0.5$ & $2.1 \pm 0.5$ & 0.53 & $<0.01^{*}$ \\
\hline \multicolumn{9}{|l|}{ BLOODS } \\
\hline \multirow[t]{2}{*}{$\mathrm{HbA}_{1 \mathrm{c}}(\% ; \mathrm{mmol} / \mathrm{mol})$} & $7.1 \pm 0.8$ & $6.9 \pm 0.7$ & $6.9 \pm 0.8$ & $7.0 \pm 0.7$ & $6.9 \pm 0.8$ & $6.6 \pm 0.9$ & 0.92 & $<0.01^{*}$ \\
\hline & $54 \pm 9$ & $52 \pm 8$ & $54 \pm 8$ & $53 \pm 8$ & $51 \pm 8$ & $49 \pm 9$ & & \\
\hline Fasting glucose (mmol/L) & $8.6 \pm 2.3$ & $8.3 \pm 1.7$ & $9.2 \pm 1.9$ & $9.5 \pm 2.3$ & $8.9 \pm 2.7$ & $8.5 \pm 2.1$ & 0.35 & 0.53 \\
\hline Triglycerides (mg/dL) & $149 \pm 82$ & $152 \pm 70$ & $161 \pm 62$ & $139 \pm 65$ & $152 \pm 93$ & $142 \pm 80$ & 0.36 & 0.17 \\
\hline C-reactive protein (mg/dL) & $7.1 \pm 10.3$ & $4.4 \pm 5.3$ & $4.7 \pm 4.3$ & $4.9 \pm 4.7$ & $3.7 \pm 4.1$ & $3.1 \pm 3.6$ & 0.33 & 0.21 \\
\hline \multicolumn{9}{|c|}{ CGM GLUCOSE CONCENTRATION } \\
\hline 24-h mean (mmol/L) & $8.4 \pm 1.4$ & $7.7 \pm 1.2$ & $8.1 \pm 1.4$ & $7.8 \pm 1.7$ & $8.4 \pm 2.1$ & $7.8 \pm 1.5$ & 0.74 & $0.01^{*}$ \\
\hline $\mathrm{SD}(\mathrm{mmol} / \mathrm{L})$ & $1.6 \pm 1.0$ & $1.3 \pm 0.5$ & $1.6 \pm 0.6$ & $1.1 \pm 0.4$ & $1.7 \pm 0.8$ & $1.5 \pm 0.7$ & 0.51 & $0.01^{*}$ \\
\hline MAGE (mmol/L) & $4.3 \pm 3.5$ & $3.1 \pm 1.3$ & $4.1 \pm 2.0$ & $2.8 \pm 1.3$ & $4.1 \pm 2.2$ & $3.7 \pm 1.6$ & 0.60 & $0.02^{*}$ \\
\hline \multicolumn{9}{|l|}{ BLOOD PRESSURE } \\
\hline Systolic (mmHg) & $130 \pm 10$ & $119 \pm 7$ & $132 \pm 13$ & $129 \pm 9$ & $128 \pm 13$ & $117 \pm 11$ & $0.03 \#$ & $<0.01^{\star}$ \\
\hline Diastolic (mmHg) & $79 \pm 6$ & $75 \pm 5$ & $83 \pm 11$ & $79 \pm 6$ & $81 \pm 7$ & $75 \pm 7$ & 0.20 & $<0.01^{*}$ \\
\hline \multicolumn{9}{|l|}{ FLOW-MEDIATED DILATION } \\
\hline Absolute FMD (mm) & $0.020 \pm 0.01$ & $0.027 \pm 0.01$ & $0.018 \pm 0.01$ & $0.024 \pm 0.01$ & $0.019 \pm 0.01$ & $0.023 \pm 0.01$ & 0.61 & $<0.01^{*}$ \\
\hline Baseline diameter (mm) & $0.41 \pm 0.10$ & $0.41 \pm 0.09$ & $0.41 \pm 0.08$ & $0.41 \pm 0.07$ & $0.41 \pm 0.07$ & $0.42 \pm 0.07$ & 0.77 & 0.71 \\
\hline Time to peak (s) & $64 \pm 26$ & $57 \pm 25$ & $60 \pm 30$ & $46 \pm 23$ & $56 \pm 21$ & $50 \pm 21$ & 0.75 & $0.05^{\star}$ \\
\hline Total energy intake (Kcal/day) & $2053 \pm 881$ & $2039 \pm 898$ & $1810 \pm 525$ & $2017 \pm 706$ & $1912 \pm 629$ & $1888 \pm 710$ & 0.35 & 0.25 \\
\hline
\end{tabular}

$H_{b A}$, Glycosylated Hemoglobin; BMI, Body Mass Index; VAT, Visceral Adipose Tissue; MAP, Mean Arterial Pressure; FMD, Flow Mediated Dilation; TE, Total Energy.

*Time effect $p<0.05$.

\#Interaction group time $p<0.05$.

change in glucose was clinically beneficial was 54\% (95\% CI: $-0.8,0.1 \mathrm{mmol} / \mathrm{L}$ ). Glycemic variability assessed by both $S D$ (by $-0.33 \pm 0.78 \mathrm{mmol} / \mathrm{L}$ ) and MAGE (by $-0.98 \pm 2.27 \mathrm{mmol} / \mathrm{L}$ ) was significantly reduced, with no differences between groups (Table 2). $\mathrm{HbA}_{1 \mathrm{c}}$ was significantly reduced after 12 weeks of HIIT (by $-0.22 \pm 0.39 \%$, Figure 3 ) with no differences between groups (Table 2). The probability that the change in $\mathrm{HbA}_{1 \mathrm{c}}$ was clinically beneficial was $0 \%$ (95\% CI: $-0.33,0.16 \%)$, with the change being most likely trivial. Fasting glucose was not significantly different after HIIT in all groups (Table 2).

\section{Body Composition}

Body mass was significantly lower after 12 weeks of HIIT (by $-0.9 \pm 3.9 \mathrm{~kg}$, Table 2), with no difference between groups. There was a significant reduction in waist circumference after 12 weeks of HIIT (by $-2.9 \pm 3.5 \mathrm{~cm}$, main effect of time: $p<$ 0.01 ) with no difference between groups (Interaction: $p=0.21$, Figure 4). Percent body fat was significantly reduced (by -0.76 $\pm 1.63 \%$, main effect of time: $p=0.02$ ) and lean body mass significantly increased (by $+1.07 \pm 2.76 \mathrm{~kg}$, main effect of time: $p=0.01$ ) after 12 weeks of HIIT, with no difference between groups (Interactions: all $p>0.83$, Figure 3 ).

\section{Cardiorespiratory Fitness $\left(\mathrm{V} \mathrm{O}_{2 \text { peak }}\right)$ and Blood Pressure}

$\dot{\mathrm{V}}{ }_{2 \text { peak }}$ significantly increased $9.8 \%$ after 12 weeks of HIIT (main effect of time: $p<0.01$, Figure 3) with no difference between groups (Interaction: $p=0.55$ ). The probability that the change in fitness was clinically beneficial was 5\% (95\% CI: 1.8, 3.1 $\mathrm{mL} / \mathrm{kg} / \mathrm{min}$ ), with the change being $95 \%$ very likely trivial.

Mean arterial blood pressure was significantly reduced after 12 weeks of HIIT (by $-5.7 \pm 7.0 \mathrm{mmHg}$, main effect of time: $p<0.01$ ) with no difference between groups (Interaction: $p=$ 0.11 , Figure 4). The probability that the change in MAP pre-post intervention was clinically beneficial was $99 \%$ (95\% CI: $-9,-2$ $\mathrm{mmHg})$.

\section{Flow-Mediated Dilation}

\%FMD significantly increased after 12 weeks of HIIT (by $+1.4 \pm$ $1.9 \%$, main effect of time: $p<0.01$ ), with no difference between groups (Interaction: $p=0.72$, Figure 4 ). The probability that the change in \%FMD was clinically beneficial was $94 \%$ likely (95\% CI: 0.86, 1.94\%). Absolute FMD also increased after HIIT (Table 2), with no difference between groups. Time to peak dilation was significantly lower (by $9.1 \pm 31.1$ s, Table 2) after 12 weeks of HIIT, with no difference between groups. Peak dilator capacity 

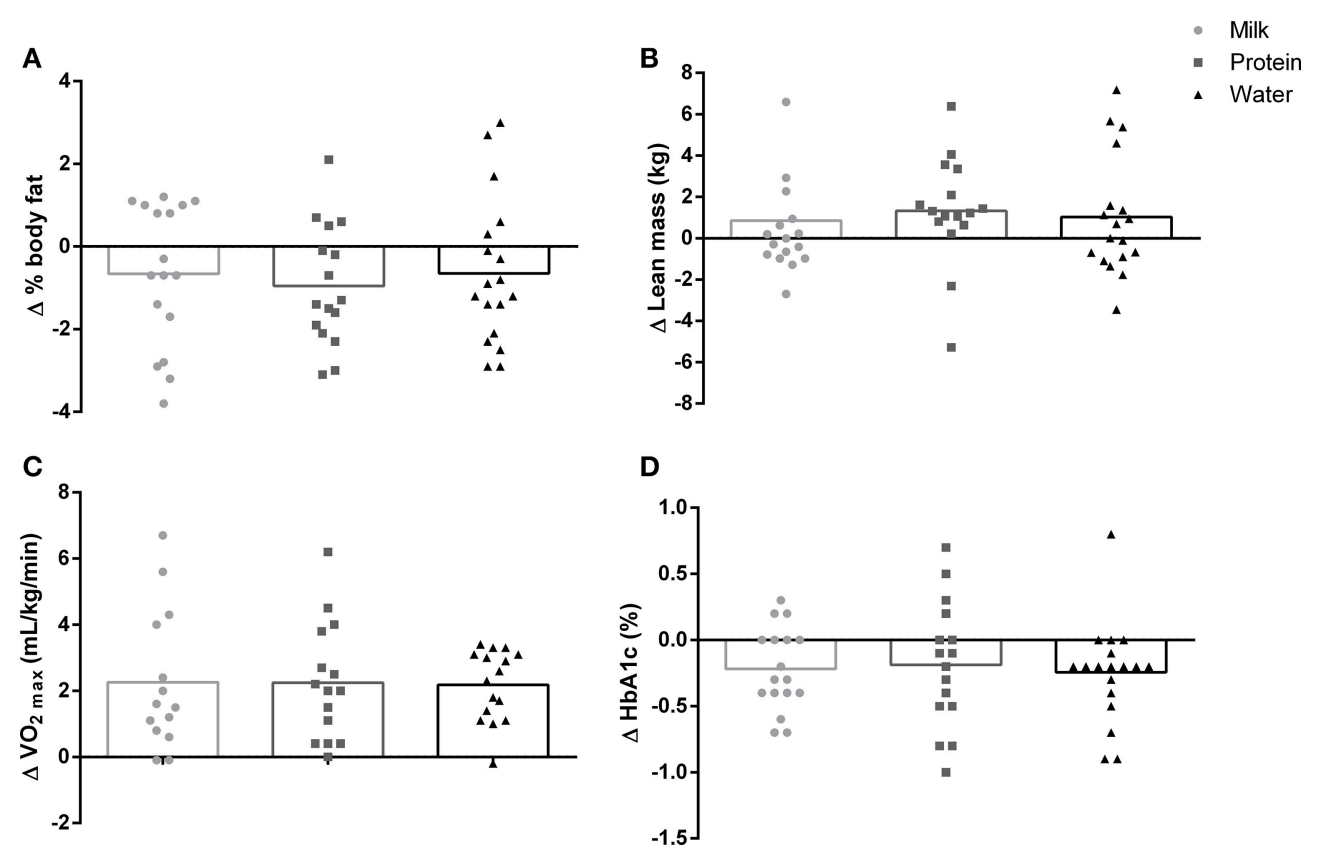

FIGURE 3 | Change from pre intervention for (A) \% body fat, (B) lean body mass, (C) cardiorespiratory fitness $\left(\dot{\mathrm{V}} \mathrm{O}_{2 \text { peak }}\right)$ and $(\mathbf{D})$ glycosylated hemoglobin $\left(\mathrm{HbA}_{1 \mathrm{C}}\right)$ in the milk, protein, and water groups (all main effect of time $p<0.05$, no group interaction $p>0.05$ ).

did not change across the intervention; Pre: $9.6 \pm 5.2 \%$, Mid: 8.1 $\pm 4.2 \%$, Post: $10.4 \pm 3.6 \%$ (main effect of time: $p=0.36$ ).

\section{Quality of Life}

PCS scores significantly increased after 12 weeks of HIIT $(n=49$, by $8.1 \pm 12.1$, main effect of time: $p<0.01$ ) with no difference between groups (Interaction: $p=0.11$ ). The probability that the change in PCS pre-post intervention was clinically beneficial was $99 \%$ likely $(95 \% \mathrm{CI}: 4.4,11.8)$. The change in MCS postintervention was different between groups ( $n=49$, Interaction: $p$ $=0.02$ ); post hoc testing revealed significant improvements in the protein group $(+12.1 \pm 9.69, p<0.01)$ but not skim-milk $(-1.1$ $\pm 13.5, p=0.79)$ or placebo $(+5.6 \pm 10.7, p=0.06)$.

\section{Dietary Intake Records}

Analysis of the 3-day diet records collected before and during the last week of the intervention showed no difference in the total daily energy intake between groups and/or across time (Table 2). Macronutrient composition of the diet was not different between groups $(p=0.32$ ), or across time: for \% carbohydrate (Pre: 48.0 $\pm 12.5 \%$ vs. Post: $48.4 \pm 13.0 \%$ of total energy, $p=0.47), \%$ protein (Pre: $20.4 \pm 4.9 \%$ vs. Post: $19.9 \pm 4.9 \%$ of total energy, $p=0.15$ ) and $\%$ fat (Pre: $30.3 \pm 12.5 \%$ vs. Post: $30.7 \pm 13.3 \%$ of total energy, $p=0.49$ ).

\section{DISCUSSION}

This study comprehensively examined the cardiometabolic benefits of HIIT in individuals with type 2 diabetes. We show for the first time that 12 weeks of low-volume HIIT, with or without post-exercise milk or protein, improves glycemic control, blood pressure, cardiorespiratory fitness, body composition, and endothelial function. Low-volume HIIT therefore appears to be a feasible and efficacious lifestyle intervention, involving minimal time and resource, to improve health in type 2 diabetes. Reducing the interval length and total exercise time has previously been shown to increase enjoyment and compliance (Martinez et al., 2015). To this end, we experienced very low dropout rates and high compliance to low-volume HIIT. In addition, we show that 12 weeks of HIIT improves quality of life, similar to previous studies in hypertensive (Molmen-Hansen et al., 2012) and heart failure (Wisløff et al., 2007) patients.

Exercise interventions generally result in modest weight loss, however exercise promotes lean mass accretion; which has important implications for whole-body metabolism, glucose disposal, and quality of life (Anton et al., 2013). Indeed, in the current study HIIT significantly increased lean mass and reduced body fat. Although weight loss was not a goal of the intervention, participants lost, on average, $\sim 0.9 \mathrm{~kg}$ of body mass, which was a statistically significant change yet small in magnitude $(\sim 1 \%)$. Generally studies report significant benefits of weight loss in the magnitude of 5-7\% (Wadden et al., 2012) but it is possible that improvements in some cardiometabollic outcomes were related to the small amount of weight loss seen. Consuming high-quality protein after exercise is known to further potentiate muscle protein synthesis (Esmarck et al., 2001; Hartman et al., 2007). Despite this, comparable changes in body composition and cardiometabolic health were seen with post-exercise milk, milk-protein, or water. In agreement, Parr et al. (2016) found changes in body composition after a combined resistance training 


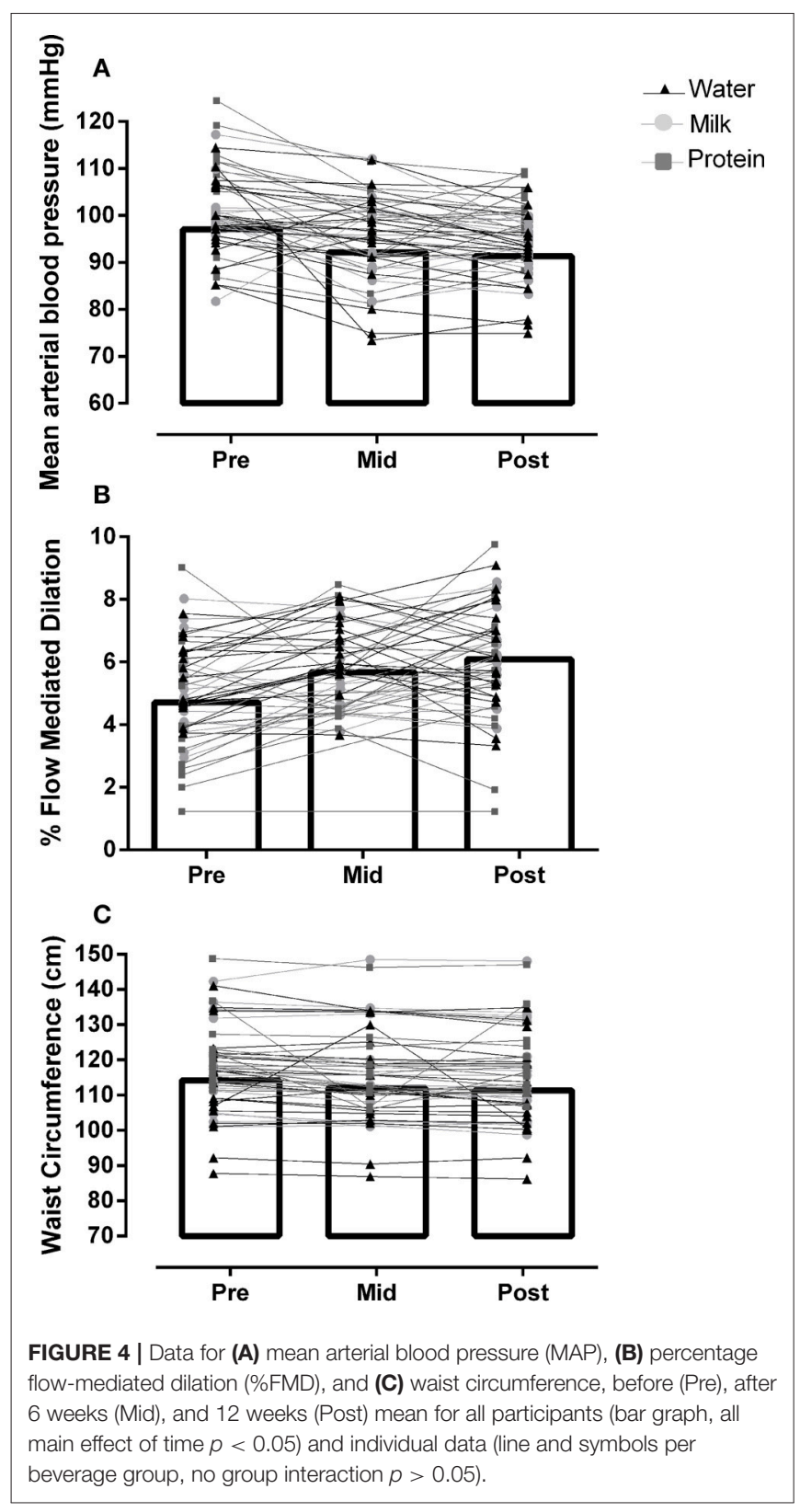

and diet intervention were independent of the amount and type of protein (high/low dairy). Epidemiological data shows an inverse relationship between low-fat dairy consumption and the risk of type 2 diabetes (Aune et al., 2013) and the addition of four servings of low fat dairy per day has been shown to improve insulin resistance (Rideout et al., 2013). Therefore, additional milk/protein supplementation (e.g., on non-exercise days) may have been needed to elucidate effects of nutritional supplementation. Indeed, some previous studies showing benefits on lean mass have provided milk/protein after exercise 5 days per week (Hartman et al., 2007; Josse et al., 2010). However, $\sim 20 \mathrm{~g}$ of post-exercise protein (similar to the current study) has been shown to maximize muscle protein synthesis (Churchward-Venne et al., 2016). To this end, a non-exercising control group may be required to detect effects of post-exercise protein added to a potent training intervention such as, HIIT. However, we feel a non-exercise control group in type 2 diabetes is unethical since numerous studies have shown worsening of glycemic control and cardiovascular risk factors in control group participants (Church et al., 2010; Karstoft et al., 2013).

Current research suggests that HIIT is more effective than continuous training for improving insulin resistance (Jelleyman et al., 2015). A recent meta-analysis revealed that absolute changes in $\mathrm{HbA}_{1 \mathrm{c}}$ are 0.5 and $0.25 \%$ greater with HIIT than control and continuous exercise, respectively (Jelleyman et al., 2015). The small, yet significant change in $\mathrm{HbA}_{1 \mathrm{c}}$ in the current study is in line with previous HIIT interventions (Madsen et al., 2015; Cassidy et al., 2016) yet robust changes in 24-h glucose were observed (Figure 2). Interestingly, the changes in 24-h glucose are similar to Karstoft et al. (2013) after 4 months of high-volume HIIT (300 min/week). This is an important finding given the perceived time barrier to exercise participation in type 2 diabetes (Korkiakangas et al., 2009). The use of CGM is a strength as it allows for additional insight into the changes in postprandial hyperglycemia and overall glycemic variability (Klonoff, 2005). Mean 24-h glucose and glycemic variability were reduced by 7 and $23 \%$, respectively, after HIIT, regardless of post-exercise nutritional supplementation. Glycemic variability may be a stronger predictor than $\mathrm{HbA}_{1 \mathrm{c}}$ for diabetes complications (Praet et al., 2006). Previous research also shows that HIIT has the potential to improve beta cell function as Madsen et al. (2015) demonstrated an increase in the oral disposition index and HOMA- $\% \beta$ after 8 weeks. The mechanisms underlying the improvements in glycemic control could not be ascertained from the present study design but likely involve a combination of improvements in peripheral insulin sensitivity, beta cell function, and hepatic insulin resistance (Karstoft et al., 2014; Madsen et al., 2015; Cassidy et al., 2016). Collectively, these findings show the potential of HIIT to improve several underlying aspects of glycemic dysfunction in type 2 diabetes.

The added benefits of vigorous exercise for cardiovascular health are well known (Marwick et al., 2009; Baldi et al., 2016) and many studies have demonstrated superior cardiovascular effects of HIIT compared to continuous training (Wisløff et al., 2007; Marwick et al., 2009; Weston et al., 2014). Extending on this work, we observed an $\sim 10 \%$ increase in cardiorespiratory fitness, a $6 \mathrm{mmHg}$ reduction in MAP and $\sim 1.4 \%$ improvement in FMD following 12 weeks of HIIT in type 2 diabetes. In itself, cardiorespiratory fitness is a strong predictor for cardiovascular mortality with each MET increase associated with a 10-20\% improvement in survival (Kodama et al., 2009). Although only a 0.7 MET increase was observed, this is in line with previous low-volume HIIT studies (Madsen et al., 2015) and participants are likely to have gained significant health benefits given their low baseline fitness $(<6 \mathrm{MET})$. A metaanalysis showed that the greatest mortality benefits occur for even small increases in fitness for those progressing from the least fit category (Kodama et al., 2009). Furthermore, the lowvolume nature of the HIIT protocol involved only 45-78 min of exercise per week with one session being resistance training. The 
combination of resistance and cardio exercise may be superior to either type alone for improving health in type 2 diabetes (Church et al., 2010). Indeed, in hypertensive patients blood pressure is reduced more with combination training than cardio alone (Lamberti et al., 2016); the 5-6 mmHg reduction is in line with the current study. Our findings suggest that HIIT performed as combined aerobic and resistance exercise clearly promotes beneficial cardiovascular adaptations in type 2 diabetes patients.

In conclusion, we show that low-volume HIIT, with or without post-exercise milk or protein supplementation, improves metabolic and cardiovascular risk factors in individuals with type 2 diabetes. The combination of resistance and aerobicbased HIIT increases lean mass, reduces fat mass, and improves endothelial function. This study, the largest and longest lowvolume HIIT study in type 2 diabetes to date, provides further evidence that HIIT is a feasible and efficacious exercise intervention to improve glycemic control, cardiovascular fitness, and body composition.

\section{REFERENCES}

Anton, S. D., Karabetian, C., Naugle, K., and Buford, T. W. (2013). Obesity and diabetes as accelerators of functional decline: can lifestyle interventions maintain functional status in high risk older adults? Exp. Gerontol. 48, 888-897. doi: 10.1016/j.exger.2013.06.007

Aune, D., Norat, T., Romundstad, P., and Vatten, L. J. (2013). Dairy products and the risk of type 2 diabetes: a systematic review and doseresponse meta-analysis of cohort studies. Am. J. Clin. Nutr. 98, 1066-1083. doi: 10.3945/ajcn.113.059030

Baldi, J. C., Wilson, G. A., Wilson, L. C., Wilkins, G. T., and Lamberts, R. R. (2016). The type 2 diabetic heart: its role in exercise intolerance and the challenge to find effective exercise interventions. Sports Med. 46, 1-13. doi: 10.1007/s40279-016-0542-9

Batterham, A. M., and Hopkins, W. G. (2006). Making meaningful inferences about magnitudes. Int. J. Sports Physiol. Perform. 1, 50-57. doi: 10.1123/ijspp.1.1.50

Borg, G. A. (1962). Physical Performance and Perceived Exertion. Lund: Gleerup Lund.

Cassidy, S., Thoma, C., Hallsworth, K., Parikh, J., Hollingsworth, K. G., Taylor, R., et al. (2016). High intensity intermittent exercise improves cardiac structure and function and reduces liver fat in patients with type 2 diabetes: a randomised controlled trial. Diabetologia 59, 56-66. doi: 10.1007/s00125-015-3741-2

Church, T. S., Blair, S. N., Cocreham, S., Johannsen, N., Johnson, W., Kramer, K., et al. (2010). Effects of aerobic and resistance training on hemoglobin A1c levels in patients with type 2 diabetes: a randomized controlled trial. JAMA 304, 2253-2262. doi: 10.1001/jama.2010.1710

Churchward-Venne, T. A., Holwerda, A. M., Phillips, S. M., and van Loon, L. J. (2016). What is the optimal amount of protein to support post-exercise skeletal muscle reconditioning in the older adult? Sports Med. 46, 1205-1212. doi: 10.1007/s40279-016-0504-2

Colberg, S. R., Sigal, R. J., Yardley, J. E., Riddell, M. C., Dunstan, D. W., Dempsey, P. C., et al. (2016). Physical activity/exercise and diabetes: a position statement of the american diabetes association. Diabetes Care 39, 2065-2079. doi: $10.2337 / \mathrm{dc} 16-1728$

Cook, N. R., Cohen, J., Hebert, P. R., Taylor, J. O., and Hennekens, C. H. (1995). Implications of small reductions in diastolic blood pressure for primary prevention. Arch. Intern. Med. 155, 701-709. doi: 10.1001/archinte.1995.00430070053006

Centers for Disease Control and Prevention (2014). National Diabetes Statistics Report: Estimates of Diabetes and its Burden in the United States, 2014. Atlanta, GA: US Department of Health and Human Services 2014.

\section{AUTHOR CONTRIBUTIONS}

MF, JL, and CD designed the study. MF, CD, KP, FH, and CC conducted the research. MF, JL, KP, and FH analyzed the data. MF and JL wrote the initial draft of the manuscript. All authors edited the manuscript and approved the final draft.

\section{FUNDING}

This study was supported by a research grant awarded to JL by the Dairy Farmers of Canada.

\section{ACKNOWLEDGMENTS}

We would like to thank all our participants for their commitment to this training study, and to our undergraduate students from UBC Okanagan Courtney, Trevor, Marcel, and Michael for their help with training. In addition thanks to Jacqueline and Jordelle from Coach Cardiology.

Esmarck, B., Andersen, J. L, Olsen, S., Richter, E. A., Mizuno, M., and KjÃęr, M. (2001). Timing of postexercise protein intake is important for muscle hypertrophy with resistance training in elderly humans. J. Physiol. 535, 301-311. doi: 10.1111/j.1469-7793.2001.00301.x

Fletcher, G. F., Ades, P. A., Kligfield, P., Arena, R., Balady, G. J., Bittner, V. A., et al. (2013). Exercise standards for testing and training a scientific statement from the American Heart Association. Circulation 128, 873-934. doi: 10.1161/CIR.0b013e31829b5b44

Francois, M. E., Durrer, C., Pistawka, K. J., Halperin, F. A., and Little, J. P. (2016). Resistance-based interval exercise acutely improves endothelial function in type 2 diabetes. Am. J. Physiol. Heart Circ. Physiol. 311, H1258-H1267. doi: 10.1152/ajpheart.00398.2016

Freedson, P. S., Melanson, E., and Sirard, J. (1998). Calibration of the Computer Science and Applications, Inc. accelerometer. Med. Sci. Sports Exerc. 30, 777-781. doi: 10.1097/00005768-199805000-00021

Godin, G., and Shephard, R. (1997). Godin leisure-time exercise questionnaire. Med. Sci. Sports Exerc. 29, 36-38.

Hartman, J. W., Tang, J. E., Wilkinson, S. B., Tarnopolsky, M. A., Lawrence, R. L., Fullerton, A. V., et al. (2007). Consumption of fat-free fluid milk after resistance exercise promotes greater lean mass accretion than does consumption of soy or carbohydrate in young, novice, male weightlifters. Am. J. Clin. Nutr. 86, 373-381. doi: 10.1016/s0162-0908(08)79232-8

Hopkins, W. G., Marshall, S. w., Batterham, A. M., and Hanin, J. (2009). Progressive statistics for studies in sports medicine and exercise science. Med. Sci. Sports Exerc. 41, 3-13. doi: 10.1249/MSS.0b013e31818cb278

Inaba, Y., Chen, J. A., and Bergmann, S. R. (2010). Prediction of future cardiovascular outcomes by flow-mediated vasodilatation of brachial artery: a meta-analysis. Int. J. Cardiovasc. Imaging 26, 631-640. doi: 10.1007/s10554-010-9616-1

Inzucchi, S. E., Bergenstal, R. M, Buse, J. B., Diamant, M., Ferrannini, E., Nauck, M., et al. (2012). Management of hyperglycaemia in type 2 diabetes: a patientcentered approach. Position statement of the American Diabetes Association (ADA) and the European Association for the Study of Diabetes (EASD). Diabetologia 55, 1577-1596. doi: 10.1007/s00125-012-2534-0

Jelleyman, C., Yates, T., O’Donovan, G., Gray, L., King, J. A., Khunti, K., et al. (2015). The effects of high-intensity interval training on glucose regulation and insulin resistance: a meta-analysis. Obesity Rev. 16, 942-961. doi: $10.1111 /$ obr.12317

Josse, A. R., Tang, J. E., Tarnopolsky, M. A., and Phillips, S. M. (2010). Body composition and strength changes in women with milk and resistance exercise. Med. Sci. Sports Exerc. 42, 1122-1130. doi: 10.1249/MSS.0b013e3181c $854 \mathrm{f} 6$ 
Karstoft, K., Winding, K., Knudsen, S. H., James, N. G., Scheel, M. M., Olesen, J., et al. (2014). Mechanisms behind the superior effects of interval vs. continuous training on glycaemic control in individuals with type 2 diabetes: a randomised controlled trial. Diabetologia 57, 2081-2093. doi: 10.1007/s00125-014-3334-5

Karstoft, K., Winding, K., Knudsen, S. H., Nielsen, J. S., Thomsen, C., Pedersen, B. K., et al. (2013). The effects of free-living interval-walking training on glycemic control, body composition, and physical fitness in type 2 diabetic patients a randomized, controlled trial. Diabetes Care 36, 228-236. doi: $10.2337 / \mathrm{dc} 12-0658$

Klonoff, D. C. (2005). Continuous glucose monitoring roadmap for 21 st century diabetes therapy. Diabetes Care 28, 1231-1239. doi: 10.2337/diacare.28. 5.1231

Kodama, S., Saito, K., Tanaka, S., Maki, M., Yachi, Y., Asumi, M., et al. (2009). Cardiorespiratory fitness as a quantitative predictor of all-cause mortality and cardiovascular events in healthy men and women: a meta-analysis. JAMA 301, 2024-2035. doi: 10.1001/jama.2009.681

Korkiakangas, E. E., Alahuhta, M. A., and Laitinen, J. H. (2009). Barriers to regular exercise among adults at high risk or diagnosed with type 2 diabetes: a systematic review. Health Promot. Int. 24, 416-427. doi: 10.1093/heapro/dap031

Lamberti, L. M., MacDonald, H. V., Johnson, B. T., Farinatti, P., Livingston, J., Zaleski, A. L., et al. (2016). Is concurrent training efficacious antihypertensive therapy? A Meta-analysis. Med. Sci. Sports Exerc. 48, 2398-2406. doi: 10.1249/MSS.0000000000001056

Lee, I.-M., Sesso, H. D., Oguma, Y., and Paffenbarger, R. S. (2003). Relative intensity of physical activity and risk of coronary heart disease. Circulation 107, 1110-1116. doi: 10.1161/01.CIR.0000052626.63602.58

Lin, X., Zhang, X., Guo, J., Roberts, C. K., McKenzie, S., Wu, W. C., et al. (2015). Effects of exercise training on cardiorespiratory fitness and biomarkers of cardiometabolic health: a systematic review and meta-analysis of randomized controlled trials. J. Am. Heart Assoc. 4:e002014. doi: 10.1161/JAHA.115.0 02014

Little, J. P., Gillen, J. B., Percival, M. E., Safdar, A., Tarnopolsky, M. A., Punthakee, Z., et al. (2011). Low-volume high-intensity interval training reduces hyperglycemia and increases muscle mitochondrial capacity in patients with type 2 diabetes. J. Appl. Physiol. 111, 1554-1560. doi: 10.1152/japplphysiol.00921.2011

Madsen, S. M., Thorup, A. C., Overgaard, K., and Jeppesen, P. B. (2015). High intensity interval training improves glycaemic control and pancreatic $\beta$ cell function of type 2 diabetes patients. PLoS ONE 10:e133286. doi: 10.1371/journal.pone.0133286

Martinez, N., Kilpatrick, M. W., Salomon, K., Jung, M. E., and Little, J. P. (2015). Affective and enjoyment responses to high-intensity interval training in overweight-to-obese and insufficiently active adults. J. Sport Exerc. Psychol. 37, 138-149. doi: 10.1123/jsep.2014-0212

Marwick, T. H., Hordern, M. D., Miller, T., Chyun, D. A., Bertoni, A. G., Blumenthal, R. S., et al. (2009). Exercise training for type 2 diabetes mellitus impact on cardiovascular risk: a scientific statement from the American Heart Association. Circulation 119, 3244-3262. doi: 10.1161/CIRCULATIONAHA.109.192521

Mazzone, T. (2010). Intensive glucose lowering and cardiovascular disease prevention in diabetes reconciling the recent clinical trial data. Circulation 122, 2201-2211. doi: 10.1161/CIRCULATIONAHA.109.913350

McHorney, C. A., WareJ. E. Jr., Lu, J. R., and Sherbourne, C. D. (1994). The MOS 36-item short-form health survey (SF-36): III. Tests of data quality, scaling assumptions, and reliability across diverse patient groups. Med. Care 32, 40-66. doi: 10.1097/00005650-199401000-00004

Molmen-Hansen, H. E., Stolen, T., Tjonna, A. E., Aamot, I. L., Ekeberg, I. S., Tyldum, G. A., et al. (2012). Aerobic interval training reduces blood pressure and improves myocardial function in hypertensive patients. Eur. J. Prev. Cardiol. 19, 151-160. doi: 10.1177/1741826711400512

Molnar, G. D., Rosevear, J. W., Ackerman, E., Gatewood, L. C., and Taylor, W. F. (1970). Mean amplitude of glycemic excursions, a measure of diabetic instability. Diabetes 19, 644-655. doi: 10.2337/diab.19.9.644

Naylor, L. H., Weisbrod, C. J., O'Driscoll, G., and Green, D. J. (2005). Measuring peripheral resistance and conduit arterial structure in humans using Doppler ultrasound. J. Appl. Physiol. 98, 2311-2315. doi: 10.1152/japplphysiol.01047.2004
Park, S. W., Goodpaster, B. H., Lee, J. S., Kuller, L. H., Boudreau, R., De Rekeneire, N., et al. (2009). Excessive loss of skeletal muscle mass in older adults with type 2 diabetes. Diabetes Care 32, 1993-1997. doi: 10.2337/dc09-0264

Parr, E. B., Coffey, V. G., Cato, L. E., Phillips, S. M., Burke, L. M., and Hawley, J. A. (2016). A randomized trial of high-dairy-protein, variable-carbohydrate diets and exercise on body composition in adults with obesity. Obesity 24, 1035-1045. doi: 10.1002/oby.21451

Praet, S. F., Manders, R. J., Meex, R. C., Lieverse, A. G, Stehouwer, C. D., Kuipers, H., et al. (2006). Glycaemic instability is an underestimated problem in Type II diabetes. Clin. Sci. 111, 119-126. doi: 10.1042/CS20060041

Reitelseder, S., Agergaard, J., Doessing, S., Helmark, I. C., Lund, P., Kristensen, N. B., et al. (2011). Whey and casein labeled with L-[1-13C] leucine and muscle protein synthesis: effect of resistance exercise and protein ingestion. Am. J. Physiol. Endocrinol. Metab. 300, E231-E242. doi: 10.1152/ajpendo.00513.2010

Rideout, T. C., Marinangeli, C. P., Martin, H., Browne, R. W., and Rempel, C. B. (2013). Consumption of low-fat dairy foods for 6 months improves insulin resistance without adversely affecting lipids or bodyweight in healthy adults: a randomized free-living cross-over study. Nutr. J. 12:56. doi: 10.1186/1475-2891-12-56

Robinson, M. M., Dasari, S., Konopka, A. R., Johnson, M. L., Manjunatha, S., Esponda, R. R., et al. (2017). Enhanced protein translation underlies improved metabolic and physical adaptations to different exercise training modes in young and old humans. Cell Metab. 25, 581-592. doi: 10.1016/j.cmet.2017.02.009

Rossetti, P., Bondia, J., Vehí, J., and Fanelli, C. G. (2010). Estimating plasma glucose from interstitial glucose: the issue of calibration algorithms in commercial continuous glucose monitoring devices. Sensors 10, 10936-10952. doi: 10.3390/s101210936

Shaw, J. E., Sicree, R. A., and Zimmet, P. Z. (2010). Global estimates of the prevalence of diabetes for 2010 and 2030. Diabetes Res. Clin. Pract. 87, 4-14. doi: 10.1016/j.diabres.2009.10.007

Tanasescu, M., Leitzmann, M. F., Rimm, E. B., Willett, W. C., Stampfer, M. J., and $\mathrm{Hu}, \mathrm{F}$. B. (2002). Exercise type and intensity in relation to coronary heart disease in men. JAMA 288, 1994-2000. doi: 10.1001/jama.288.16.1994

Thijssen, D. H., Black, M. A., Pyke, K. E., Padilla, J., Atkinson, G., Harris, R. A., et al. (2011). Assessment of flow-mediated dilation in humans: a methodological and physiological guideline. Am. J. Physiol. Heart Circ. Physiol. 300, H2-H12. doi: 10.1152/ajpheart.00471.2010

Thompson, P. D., Arena, R., Riebe, D., and Pescatello, L. S. (2013). ACSM's new preparticipation health screening recommendations from ACSM's guidelines for exercise testing and prescription. Curr. Sports Med. Rep. 12, 215-217. doi: 10.1249/JSR.0b013e31829a68cf

Tinken, T. M., Thijssen, D. H., Hopkins, N., Dawson, E. A., Cable, N. T., and Green, D. J. (2010). Shear stress mediates endothelial adaptations to exercise training in humans. Hypertension 55, 312-318. doi: 10.1161/HYPERTENSIONAHA.109.146282

UK Prospective Diabetes Study Group (1998). Tight blood pressure control and risk of macrovascular and microvascular complications in type 2 diabetes: UKPDS 38. BMJ 317, 703-713. doi: 10.1136/bmj.317.7160.703

Wadden, T. A., Webb, V. L., Moran, C. H., and Bailer, B. A. (2012). Lifestyle modification for obesity. Circulation 125, 1157-1170. doi: 10.1161/CIRCULATIONAHA.111.039453

Warkentin, L. M, Das, D., Majumdar, S. R, Johnson, J. A, and Padwal, R. S (2014). The effect of weight loss on health-related quality of life: systematic review and meta-analysis of randomized trials. Obes. Rev. 15, 169-182. doi: $10.1111 /$ obr.12113

Weston, K. S., Wisløff, U., and Coombes, J. S. (2014). High-intensity interval training in patients with lifestyle-induced cardiometabolic disease: a systematic review and meta-analysis. Br. J. Sports Med. 48, 1227-1234. doi: 10.1136/bjsports-2013-092576

WHO Expert Consultation (2008). Waist Circumference and Waist-Hip Ratio. Report of a WHO Expert Consultation. Geneva: World Health Organization, 8-11.

Wing, R. R, Bolin, P., Brancati, F. L, Bray, G. A, Clark, J., Coday, M., et al. (2013). Cardiovascular effects of intensive lifestyle intervention in type 2 diabetes. $N$. Engl. J. Med. 369, 145-154. doi: 10.1056/NEJMoa1212914

Wisløff, U., Støylen, A., Loennechen, J. P., Bruvold, M., Rognmo,Ø., Haram, P. M., et al. (2007). Superior cardiovascular effect of aerobic interval training 
versus moderate continuous training in heart failure patients a randomized study. Circulation 115, 3086-3094. doi: 10.1161/CIRCULATIONAHA.106.6 75041

Woodman, R. J, Playford, D. A, Watts, G. F, Cheetham, C., Reed, C., Taylor, R. R, et al. (2001). Improved analysis of brachial artery ultrasound using a novel edge-detection software system. J. Appl. Physiol. 91, 929-937.

Yeboah, J., Crouse, J. R., Hsu, F. C., Burke, G. L., and Herrington, D. M. (2007). Brachial flow-mediated dilation predicts incident cardiovascular events in older adults the cardiovascular health study. Circulation 115, 2390-2397. doi: 10.1161/CIRCULATIONAHA.106.678276
Conflict of Interest Statement: The authors declare that the research was conducted in the absence of any commercial or financial relationships that could be construed as a potential conflict of interest.

Copyright (๑ 2017 Francois, Durrer, Pistawka, Halperin, Chang and Little. This is an open-access article distributed under the terms of the Creative Commons Attribution License (CC BY). The use, distribution or reproduction in other forums is permitted, provided the original author(s) or licensor are credited and that the original publication in this journal is cited, in accordance with accepted academic practice. No use, distribution or reproduction is permitted which does not comply with these terms. 